\title{
Effect of crosslinking on the microtribological behavior of model polymer brushes
}

Article

Accepted Version

Singh, M. K., Ilg, P., Espinosa-Marzal, R. M., Kroger, M. and Spencer, N. D. (2016) Effect of crosslinking on the microtribological behavior of model polymer brushes. Tribology Letters, 63 (2). 17. ISSN 1573-2711 doi:

https://doi.org/10.1007/s11249-016-0705-8 Available at https://centaur.reading.ac.uk/65953/

It is advisable to refer to the publisher's version if you intend to cite from the work. See Guidance on citing.

Published version at: http://link.springer.com/article/10.1007/s11249-016-0705-8

To link to this article DOI: http://dx.doi.org/10.1007/s11249-016-0705-8

Publisher: Springer

All outputs in CentAUR are protected by Intellectual Property Rights law, including copyright law. Copyright and IPR is retained by the creators or other copyright holders. Terms and conditions for use of this material are defined in the End User Agreement.

www.reading.ac.uk/centaur 
Central Archive at the University of Reading

Reading's research outputs online 


\title{
Effect of Crosslinking on the Microtribological Behavior of Model Polymer Brushes
}

\author{
Manjesh K. Singh ${ }^{1}$, Patrick Ilg², Rosa M. Espinosa-Marzal ${ }^{1,3}$, \\ Martin Kröger ${ }^{4}$, Nicholas D. Spencer ${ }^{*}$
}

${ }^{1}$ Laboratory for Surface Science and Technology, Department of Materials, ETH Zurich, 8093 Zurich, Switzerland.

${ }^{2}$ School of Mathematical and Physical Sciences, University of Reading, Reading RG6 6AX, United Kingdom.

${ }^{3}$ Laboratory for Smart Interfaces in Environmental Nanotechnology, Department of Civil and Environmental Engineering, University of Illinois at Urbana-Champaign, Champaign, Illinois 61801, United States.

${ }^{4}$ Polymer Physics, Department of Materials, ETH Zurich, 8093 Zurich, Switzerland.

*Corresponding Author

E-mail: nspencer@ethz.ch, Phone: +41-44-632-5850,Fax: +41-44-633-1027

KEYWORDS: tribology; boundary lubrication; molecular dynamics; polymer brushes, crosslinking

ABSTRACT: Polymer brushes in good solvents are known to exhibit excellent tribological properties. We have modeled polymer brushes and their gels using a multibead-spring model and studied their tribological behavior via nonequilibrium molecular-dynamics (MD) simulations. Simulations of brushagainst-wall systems were performed using an implicit solvent-based approach. Polymer chains were modeled as linear chains, randomly grafted on a planar surface. Quantities extracted from the simulations are the normal stress, shear stress and concentration profiles. We find that while an increase in the degree of crosslinking leads to an increase in the coefficient of friction, an increase of the length of crosslinker chains does the opposite. Effect of crosslinking can be understood in two ways: (i) there are fewer polymer chains in the outer layer as the degree of crosslinking increases to take part in brush-assisted lubrication, and (ii) crosslinked polymer chains are more resistant to shear than non-crosslinked ones.

INTRODUCTION: When polymer chains are grafted on a surface at a grafting density such that the distance between grafting points is less than the radius of gyration $\left(R_{g}\right)$ of an unperturbed single chain, they stretch out in the presence of a good solvent to avoid segment-segment interaction, forming a brush-like structure. The interactions among tethered chains result in a strong osmotic repulsive force in a good solvent, implying interesting interfacial features such as lubrication and antifouling properties. Polymer-brush-coated surfaces find applications in diverse fields including colloidal stabilization, adhesion, biocompatibility and tribology.[1-5] Crosslinked polymer brushes are called polymer brush- 
gels or simply gels. Such gels have many applications in the fields of tissue engineering and biotribology.[6, 7]

Shearing hard surfaces against each other leads to asperity-asperity contacts, resulting in a high value of the coefficient of friction at the contact surface. In contrast, when surfaces bearing polymer brushes are sheared against each other in the presence of a good solvent, very low coefficients of friction are observed.[8, 9] A strong repulsive force of entropic origin prevents interpenetration of polymer chains even at high compressions, enabling the development of a thin fluid film between opposing brushes;[10] similarly, in brush-against-wall systems the repulsive force prevents contact with the counter-surface. This thin fluid film assists in reducing friction at the contact. When surfaces bearing polymer gels are brought in contact (or gel-against-wall), the role of brush forming polymer chains in the outermost layer that assist in brush-mediated lubrication is significant. Upon increasing the degree of crosslinking, the number of chains available for the formation of a highly solvated brush architecture in the outer layer diminishes, leading to a decrease in lubricious behavior.[11, 12]

Tribological properties of polymer-brush-based lubricants have long been studied under different loads and speeds. Boundary and hydrodynamic regimes have been described.[13] Both experimental[10, 1418] and simulation studies[19-28] have been carried out to understand the tribological behavior of polymer brushes in solvents of different qualities. Experiments have been carried out to study the tribological behavior of polymer brushes at the nano, micro and macro scales. Nalam et al.[29] studied PLL-g-dextran and PLL-g-PEG polymer brushes in different mixtures of solvent at the nano scale employing the surface forces apparatus (SFA) and colloidal-probe lateral force microscopy (LFM) at different loads and speeds; a nanoscale Stribeck curve was established.

There has been also great interest in the tribological behavior of gels.[7, 11, 12, 30-35] Pan et al.[31] studied the friction properties of poly(vinyl alcohol) hydrogels against titanium alloys for their biotribological application under various loads and speeds and concluded that the effect of load on friction was greater than that of the speed. Poly(2-hydroxyethyl methacrylate) (PHEMA) hydrogels have also been of special interest for researchers for their application in biotribology and studies have been carried out for different combinations of substrate and counter-surface.[7, 30, 36, 37] Li et al.[11] studied the effect of degree of crosslinking on mechanical and tribological behavior of poly(acrylamide) (PAAM) brushes and hydrogels. They found that covalently crosslinked hydrogels displayed higher Young's moduli and coefficients of friction in comparison to surface-grafted polymer brushes and the effect was found to increase with degree of crosslinking. In contrast, Ishikawa et al.[38] compared the effect of mechanical properties and of chemical characteristics (polymer hydration) on tribological behavior of hydrogels via pin-on disk experiments, and concluded that the chemical characteristics (e.g. hydration) were the dominant factors. Ohsedo et al.[37] studied the effect of presence of well-defined polymer brushes on gel surfaces. Their study showed that longer poly (sodium 4-styrenesulfonate) (PNaSS) brushes on PHEMA gels exhibit lower friction at low sliding speeds. Dunn et al.[6] explored the distinction between a self-mated "Gemini" hydrogel interface and hydrogels sliding against hard, impermeable counter-surfaces and demonstrated that Gemini interfaces have very low friction coefficients independently of sliding speed, whereas hydrogels against rigid impermeable surfaces exhibit higher friction, which is strongly dependent on sliding speed or time in 
contact. Thus, experimental studies have mainly focused on the role of solvent and effect of degree of crosslinking on tribological behavior of gels but to the best of our knowledge there is no study of the role of length of crosslinkers.

The first theoretical studies of polymer brushes on flat surfaces were performed by Alexander,[39] de Gennes[40] and Semenov[41]. Alexander and de Gennes assumed a 'step-like' density profile, while Milner et al.[42] later suggested a parabolic density profile in their work. The concept of strong stretching of brushes was implemented by Milner et al.[42] and Zhulina et al.[43] independently, implying that the brush height is much larger than an unperturbed extension of a single chain. Simulation work to understand polymer brush behavior has been carried out using various approaches such as Monte Carlo[44-46], Brownian dynamics[47] and dissipative particle dynamics[8]. Several molecular-dynamics simulation studies have been also carried out to understand the details of polymerbrush-based tribology using both implicit[20, 21, 25-27] and explicit solvents[22, 48-56]. Prior to Galuschko et al. [28, 57] there were not many molecular-dynamics studies comparing implicit-solventand explicit-solvent-based approaches. Simulation studies have focused on different thermostatting approaches, and a comparison of Langevin dynamics and dissipative dynamics methods has been published[58]. Most of the implicit-solvent-based studies have considered a Langevin-dynamics approach. Simulation studies on gels mostly focused on conformations and solvent-gel interactions[5963]. To the best of our knowledge no simulation studies of the tribological behavior of gels have been reported.

MODEL: We performed computer simulation studies to facilitate the understanding of the tribological behavior of polymer gels at a molecular scale. The simulations were carried out for a multibead-springmodel-based, coarse-grained molecular-dynamics technique using the open source code LAMMPS (Large-scale Atomic/Molecular Massively Parallel Simulator).[64] Attractive interactions between bonded beads within linear polymer chains were modeled using the finitely extendable nonlinear elastic (FENE) potential. Van der Waals interactions between all beads in the system were modeled using the classical Lennard-Jones (LJ) 12-6 potential. In addition we used a repulsive LJ 9-3 potential between beads and grafting surface to ensure the polymer chains do not cross the grafting surface. The details of our polymer brush modeling can be found in previously published work, where we also discuss how to map from dimensionless Lennard-Jones units to real units for a particular system under study.[27]

Crosslinking between chains in polymer-brush systems was performed using the following approach. After the polymer-brush system was generated via a growth procedure,[65] uncrosslinked pairs of beads separated by a distance that was compatible with the extension of a crosslinker chain were randomly selected for crosslinking. A crosslinker chain was inserted if it did not overlap with existing beads. This was repeated until the desired number of crosslinker chains had been inserted. 


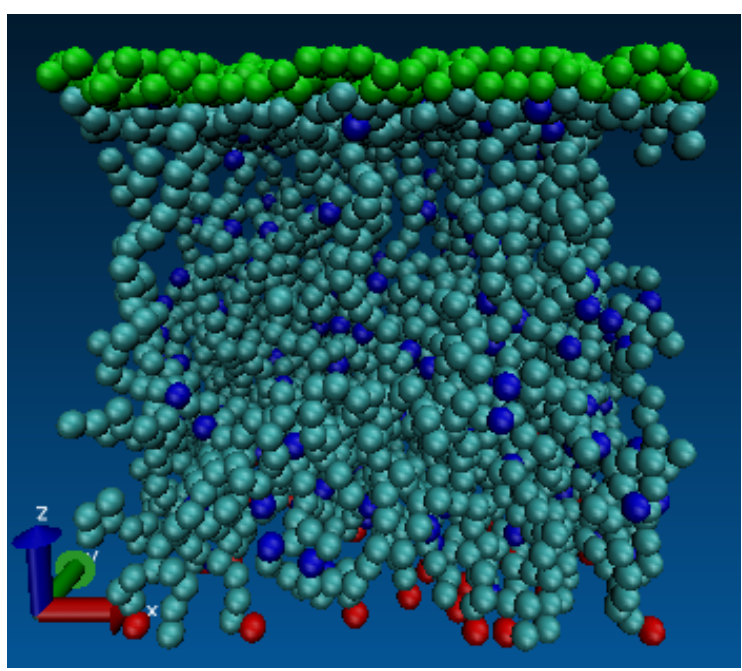

Figure 1: Schematics of the crosslinked polymer brush in the presence of an opposing wall. Redcolored beads are tethered, cyan beads constitute the polymer chains, blue beads are crosslinkers of length $L S=2$ and green beads represent the explicit wall.

The crosslinked polymer-brush system was generated for different numbers of crosslinkers/spacers (NS) for a fixed contour length of crosslinker/spacer (LS) chains and vice-versa. Figure 2a shows two crosslinked chains in a crosslinked polymer brush. Figure $2 \mathrm{~b}$ shows the explicit crosslinkers, where LS $=1$ means that there is no explicit bead in a crosslinker but that it is represented by a single bond between crosslinked beads, LS $=2$ means that the explicit crosslinker chain has a single interior bead which is bonded to two beads in the respective chains to be crosslinked and similarly a LS $=4$ explicit crosslinker has 3 interior beads which are bonded to form a linear chain and each end is bonded to a bead in the crosslinked chains. For bonding within crosslinkers and bonding between crosslinkers and chains in the polymer brush system we have used a harmonic bond potential,

$$
E_{H}=K_{H}\left(r-r_{0}\right)^{2}
$$

where $K_{H}$ is the bond strength, $r_{0}$ the equilibrium bond length and $r$ is the distance between bonded atoms at any given time. We used typical values $K_{H}=100$ and $r_{0}=1$ (all the quantities are in reduced Lennard-Jones unit).

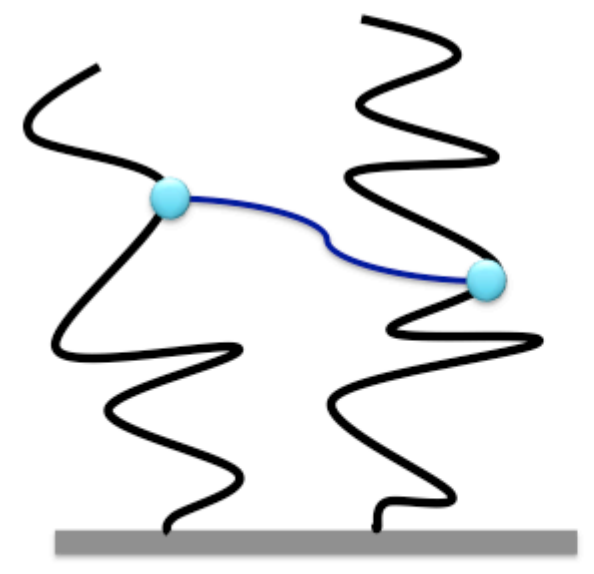

(a)

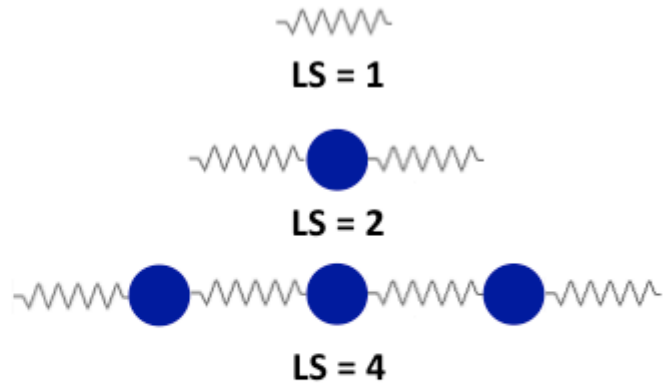

(b)

Figure 2: Crosslinking and crosslinkers. Schematic drawing showing (a) a crosslinker chain between two crosslinked chains, and (b) crosslinker chains with different equilibrium lengths. 
Good solvent quality was modeled implicitly by incorporating only the repulsive part of the LJ/12-6 interaction between non-bonded pairs (WCA potential). The dynamics of such a molecular model at constant temperature were explored by solving modified (thermostatted) Newton's equations with an implicit solvent (Langevin equations). The calculated chain trajectories and their forces allow us to extract chain orientation, density profiles, and the stress tensor. In a first step, the polymer-brushbearing surface and the wall were brought near to each other and allowed to equilibrate. In a second step, tethered beads were moved at constant shear speed, maintaining the separation between the grafting surface and the wall constant. Normal and shear stresses were calculated using the IrvingKirkwood expression for the stress tensor and the coefficient of friction was determined as the ratio between shear and normal stress-tensor components. The corresponding results allowed us to shed light on polymer-brush systems including semiflexible and cross-linked chains, which had so far not been investigated in detail.

All the simulations were carried for the brush-against-wall system (Fig. 1). The LJ-12/6 potential with a cut-off of $R_{\mathrm{cw}}=2.5$ was used for interactions between polymer chains and explicit-wall beads in the counter surface. The brush-against-wall system has $M=50$ chains tethered randomly on the grafting surface, each chain consisting of $N=50$ beads. It is to be noted that the critical grafting density[28] $\left(\rho^{*}\right.$ $=1 / \pi R_{\mathrm{g}}{ }^{2}$ ) for chains having $N=50$ beads is $\rho^{*} \approx 0.015$. We have considered a grafting density of $\rho=$ 0.075 , which is 5 times larger than the critical grafting density. The explicit wall counter-surface was made of beads residing on a regular lattice parallel to the tethering surface, but randomly placed at altitudes $(D-\Delta), D$ being the distance from the grafting surface and $\Delta$ is randomly chosen as $0.5,1.0$ or 1.5 to mimic the effect of roughness. Simulations were performed for systems with different lengths of crosslinker chains (LS) and numbers of crosslinker chains (NS), as summarized in Table 1.

Table 1: Simulations carried out for $M=50$ chains of $N=50$ beads each, subject to the following lengths of crosslinker chains (LS) and amount of crosslinker chains (NS), where (100\%) means that each monomer is connected to exactly one crosslinker chain.

\begin{tabular}{|c|c|c|c|}
\hline LS & \multicolumn{3}{|c|}{ NS (Degree of Crosslinking in \%) } \\
\hline 1 & $50(4 \%)$ & $100(8 \%)$ & $200(16 \%)$ \\
\hline 2 & $50(4 \%)$ & $100(8 \%)$ & $200(16 \%)$ \\
\hline 4 & $50(4 \%)$ & $100(8 \%)$ & $200(16 \%)$ \\
\hline
\end{tabular}

The speed-dependent studies were carried out by maintaining the separation $D$ between the grafting surface and the explicit wall constant at $D=8$. We set the surfaces in relative motion to each other in order to simulate tribological properties. Simulations were carried out for various fixed lateral speeds $S$ $=0.0003,0.0005,0.0007,0.001$ and 0.003 of the tethered beads, while the opposing rough wall was kept in place at a distance $D=8$, corresponding to an applied shear rate of $\dot{\gamma}=S / D$. The simulations were carried out for a duration of 90,500,000 time steps (data for first 30,500,000 steps being ignored in the analysis) at speed $S=0.0003$ and for 9,500,000 steps (ignoring data for first 3,500,000 steps) for speed $S=0.003$, where the time step was chosen as $\Delta t=0.002$ when data was ignored until steady state 
was reached and $\Delta t=0.0025$ when data was recorded and analyzed. The Langevin thermostat parameters used were $T=1.2$ and damping coefficient $\xi=0.1$.

\section{EFFECT OF NUMBER OF CROSSLINKERS (NS)}

Figure 3 shows results highlighting the effect of degree of crosslinking for crosslinkers of length LS = 1 on the (a) normal stress, (b) shear stress, (c) coefficient of friction against speed, and (d) volume fraction of polymer against separation $D$. The normal stress is found to be constant over the speeds studied for all the systems under consideration whereas it is found to be decreasing with an increase in the degree of crosslinking. The shear stress increases with increasing speed for all the systems and it also increases with an increase in the degree of crosslinking. The coefficient of friction we find to increase with an increase in the degree of crosslinking. This is consistent with previous experimental observations[11].

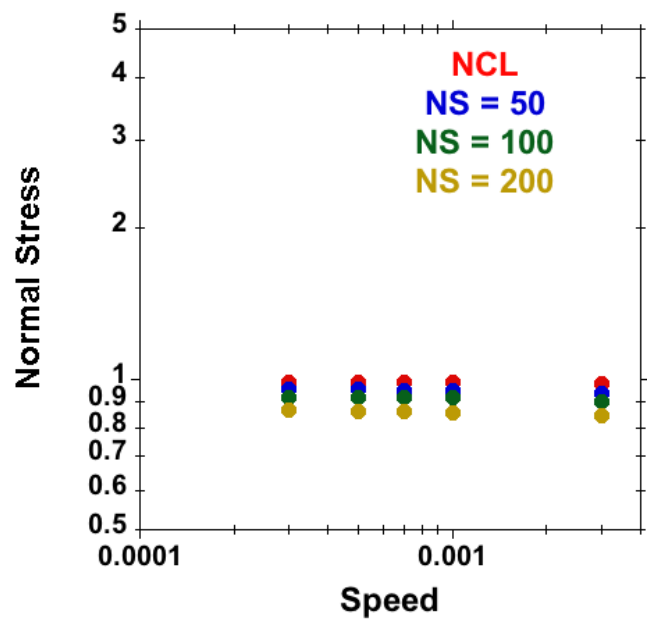

(a)

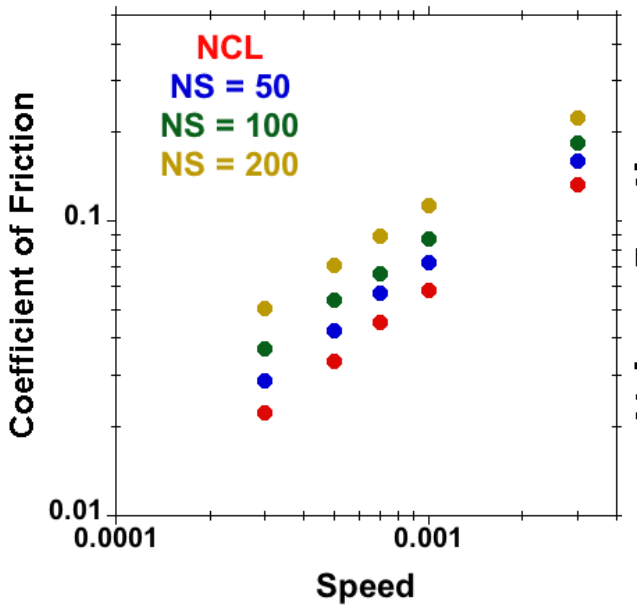

(c)

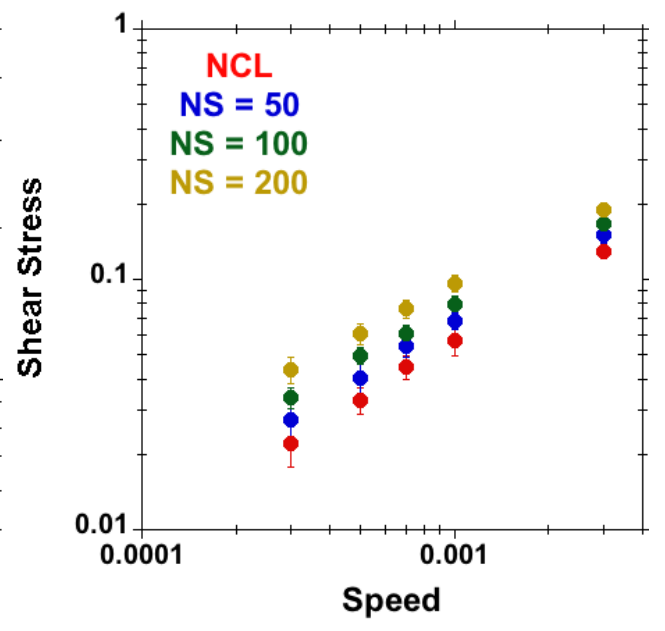

(b)

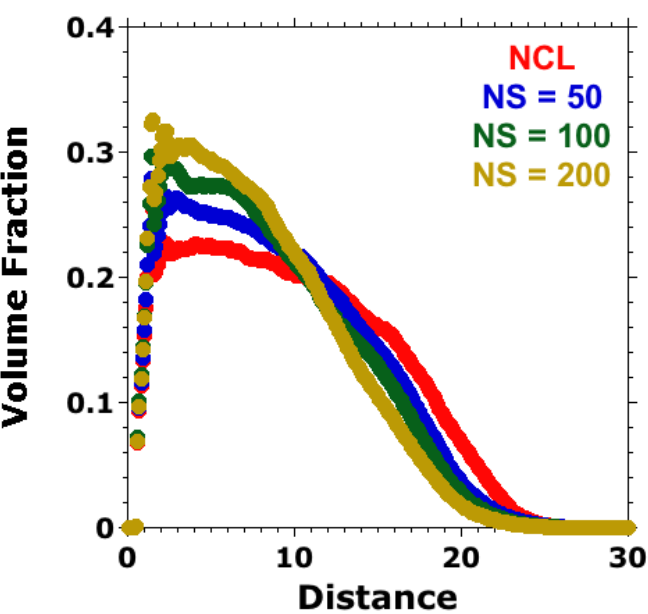

(d)

Figure 3: Effect of degree of crosslinking at constant length of crosslinkers, LS =1, except for the noncrosslinked (NCL) brush. (a) Normal stress against shear speed, (b) shear stress against speed, (c) coefficient of friction against speed, and (d) volume fraction against distance from the tethering surface for systems consisting of $M=50$ chains with $N=50$, at grafting density $\rho=0.075$, length of crosslinkers/spacers $L S=1$, and for different numbers of spacers/crosslinkers (NS) $=0$ (NCL), 50, 100 and 200. The density profile is shown for a separation of $D=30$ between grafting surface and wall, at vanishing speed, $S=0$. 
Figure 3(d) shows density profiles for polymer-brush systems with different degrees of crosslinking for the shortest (single bond) crosslinkers $(\mathrm{LS}=1)$ in terms of volume fraction of polymers against distance. The decrease in normal stress with an increase in the degree of crosslinking in comparison to uncrosslinked polymer brushes (NCL) can be explained with the help of the recorded density profile. The brush height decreases with increasing degree of crosslinking, so less compression is experienced by brushes with a higher degree of crosslinking at the same separation between wall and the polymerbearing surface. This result in a decrease of the normal stress applied at the same separation with increasing degree of crosslinking. The increase in shear stress with increase in degree of crosslinking can also be explained with the help of the density profile. As the degree of crosslinking increases, the more collapsed configuration of the brush leads to fewer polymer chains being available in the outer layer to assist brush-mediated lubrication.[5, 9, 11, 18] The lateral motion of the polymer chains also becomes more inter-dependent, which results in an increase in the shear stress or coefficient of friction upon increasing the degree of crosslinking.

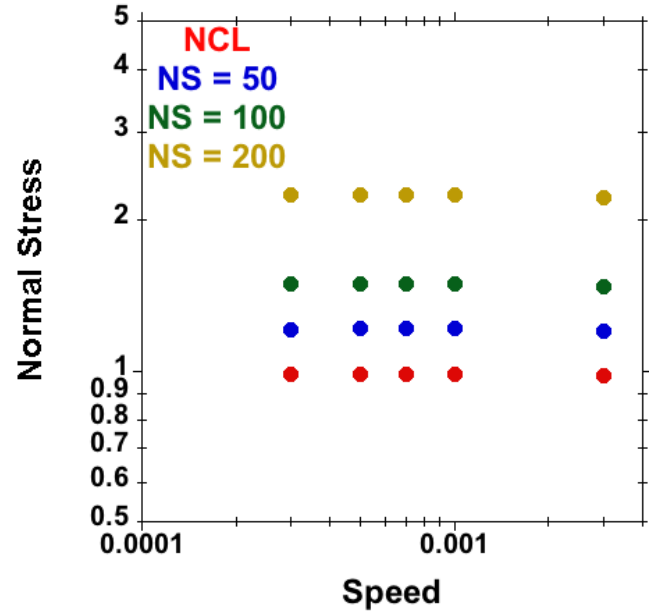

(a)

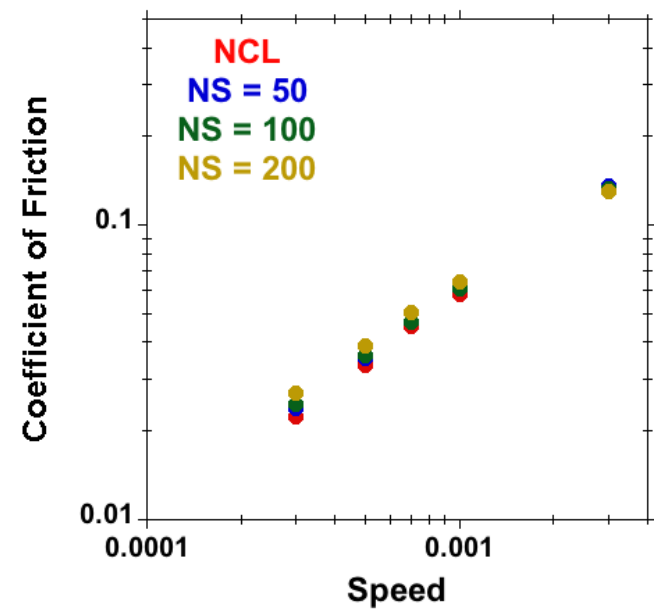

(c)

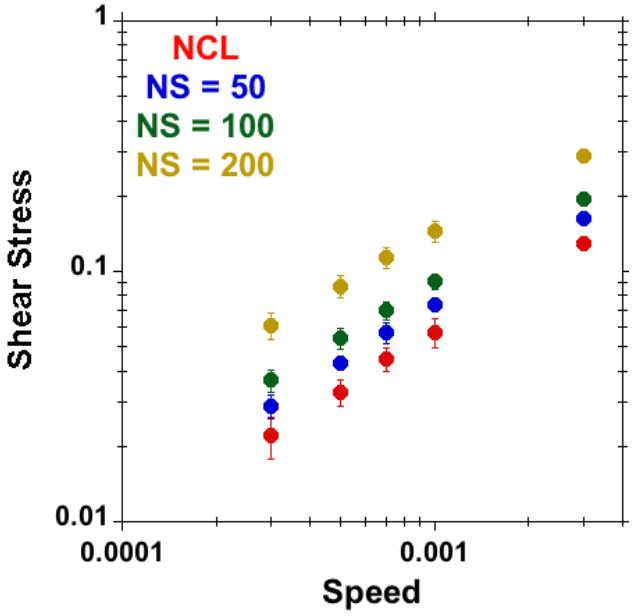

(b)

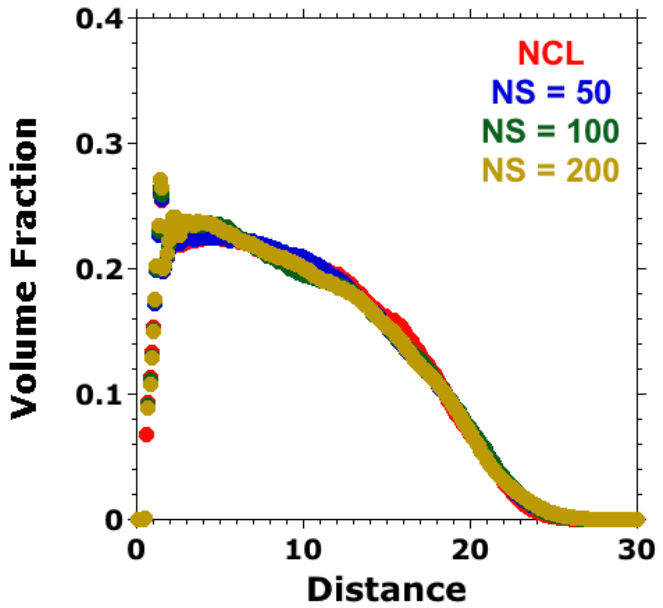

(d)

Figure 4: Effect of the degree of crosslinking at constant length of crosslinker chains, LS = 4. (a) Normal stress against shear speed, (b) shear stress against speed, (c) coefficient of friction against speed, and (d) volume fraction against distance for systems made of $M=50, N=50, \rho=0.075, L S=4$ and for different number of spacers/crosslinkers (NS) $=0$ (NCL), 50, 100 and 200. The volume 
fraction profile is plotted for a separation of $D=30$ between grafting surface and wall, at vanishing speed, $S=0$.

Figure 4 presents the the effect of degree of crosslinking on polymer-brush systems having crosslinkers of length LS=4 in terms of (a) normal stress against speed, (b) shear stress against speed, (c) coefficient of friction against speed, and (d) volume fraction against distance. The normal stress is found to remain constant over the speeds studied for each system, whereas it increases with increasing degree of crosslinking at every speed. The shear stress is found to be increasing with speed for each of the systems studied and also increases with increasing degree of crosslinking at each speed. The coefficient of friction we find to be increasing with increasing speed for each system and to also increase with increasing degree of crosslinking at given speed. However, the effect of the degree of crosslinking on the coefficient of friction for polymer-brush systems with $\mathrm{LS}=4$ is not as significant as was observed with shorter crosslinkers, $L S=1$.

The observations can be rationalized with the help of the density profiles for the different systems. The density profiles and brush heights for systems with different degrees of crosslinking at LS=4 are quite similar to the corresponding uncrosslinked polymer brush systems. The increase in normal and shear stress with increase in the degree of crosslinking can be explained by an increase in the number density (due to the additional beads in the crosslinker chains) upon increasing the degree of crosslinking (note that the volume fractions in Figures 3-6 does not include the crosslinker beads). The coefficient of friction (ratio of shear stress and normal stress) exhibits a slight increase with increase in degree of crosslinking at each speed, which is consistent with the density profiles for the different degrees of crosslinking at $\mathrm{LS}=4$ in comparison with the uncrosslinked polymer-brush systems. The slight increase in the coefficient of friction upon increasing the degree of crosslinking can be attributed to the increased restriction of the interdependent lateral motion with an increase in the degree of crosslinking. Uncrosslinked polymer chains are deformed easily in comparison to gels under shear.[12, 32, 37]

\section{EFFECT OF LENGTH OF CROSSLINKERS (LS)}

Figure 5 presents the results for the crosslinked polymer brush systems with $\mathrm{NS}=50$ crosslinkers/spacers, to facilitate the study of the effect of length of crosslinkers (LS) in terms of (a) normal stress against speed, (b) shear stress against speed, (c) coefficient of friction against speed, and (d) volume fraction against distance. The normal stress was found to remain constant for each of the systems over the speeds studied. In comparison to the uncrosslinked systems, the normal stresses at each speed decreased for LS=1 but they increased with increasing length of crosslinkers for LS=2 and $\mathrm{LS}=4$. The shear stress for all the crosslinked systems was found to be higher than that of the uncrosslinked systems, but no clear effect of the length of crosslinkers is observed for any of the systems. The shear stress is found to be increasing with an increase in speed for all the brush systems. The coefficient of friction is found to be increasing with increase in speed for each LS whereas the coefficient of friction slightly decreases with an increase of the length of crosslinkers at all speeds studied.

The decrease in normal stress for systems with LS $=1$ crosslinkers in comparison to uncrosslinked polymers can be explained by a decrease in brush height with increasing degree of crosslinking for short LS $=1$ crosslinkers. As the brush height is smaller for the crosslinked brushes with LS $=1$, they 
are less compressed in comparison to uncrosslinked polymer brushes (NCL) at identical separation when separation (D) between the surfaces is decreased. As the length of crosslinkers increases (LS $=2$ and LS =4), the brush height of the system increases and is almost similar to the uncrosslinked (NCL) polymer brush system. The shear stress for crosslinked polymer brushes was higher than that of uncrosslinked polymer brushes because crosslinked chains offer more resistance to shear. The coefficient of friction vs speed curve is consistent with the trend highlighted by the volume fraction profiles. The profiles of crosslinked brushes with longest crosslinkers are basically indistinguishable from those of uncrosslinked brushes, which is reflected in their similar coefficients of friction.

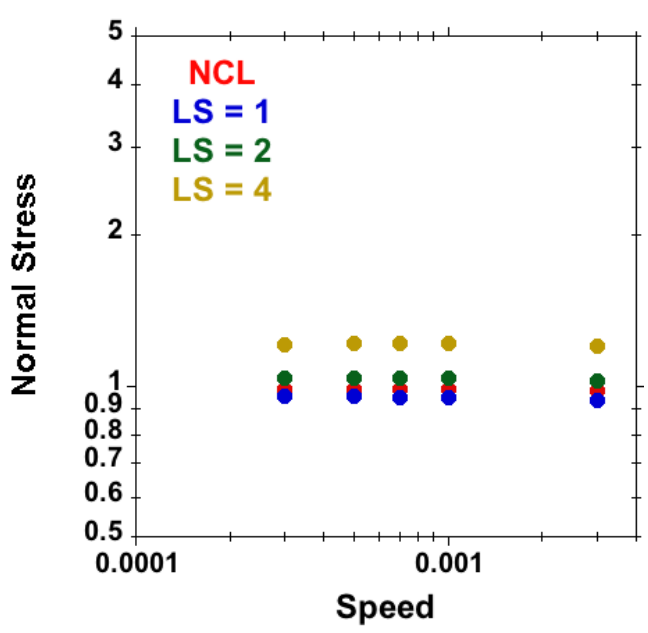

(a)

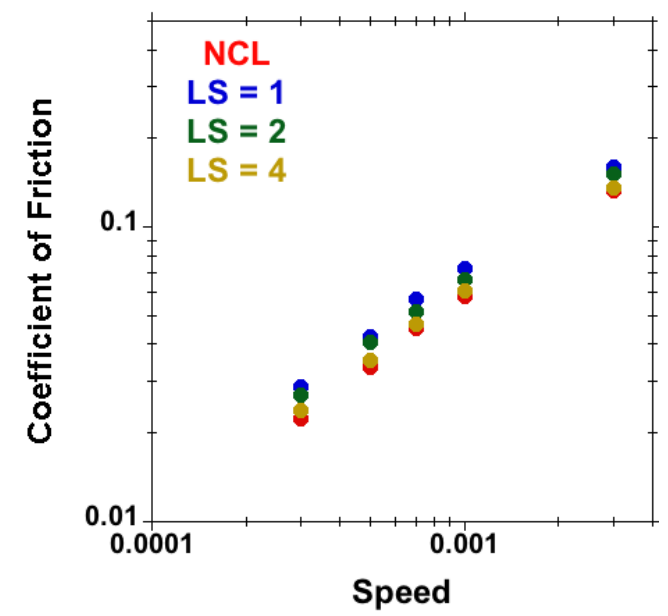

(c)

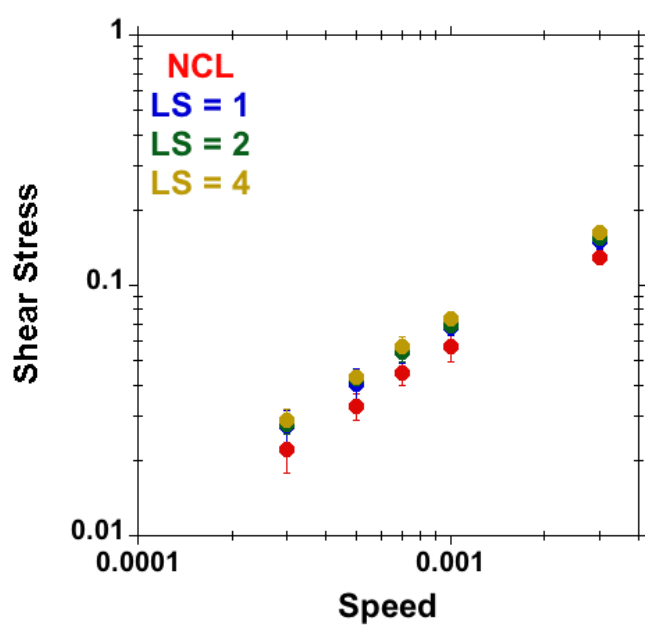

(b)

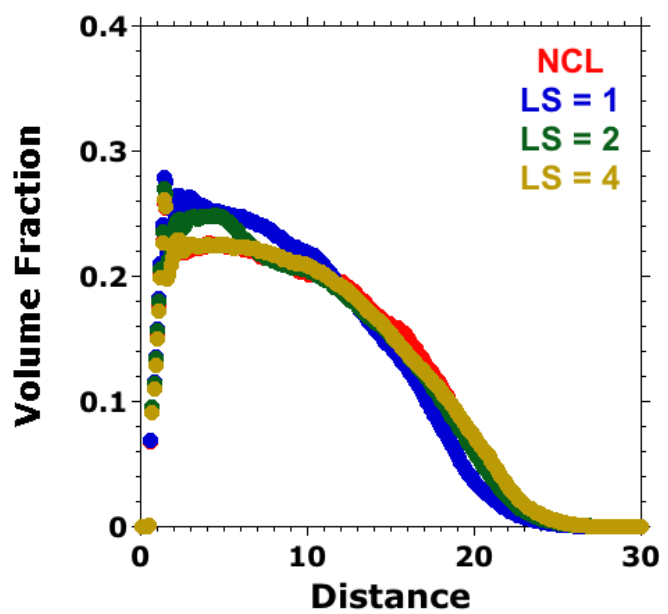

(d)

Figure 5: Effect of length of crosslinkers at constant degree/number of crosslinker chains, NS = 50 . (a) Normal stress against speed, (b) shear stress against speed, (c) coefficient of friction against speed, and (d) volume fraction against distance for $M=50, N=50, \rho=0.075, \mathrm{NS}=50$ and for different lengths of spacers/crosslinkers $(L S)=0(N C L), 1,2$ and 4 . The volume fraction profile is plotted for a separation of $D=30$ between grafting surface and wall, at vanishing speed, $S=0$.

Figure 6 shows results for uncrosslinked and crosslinked polymer brushes with NS $=200$, to facilitate the study of the effect of length of crosslinkers in terms of normal and shear stresses, and coefficient of friction against speed, and the volume fraction against distance to the grafting surface. The normal stress here also decreased for LS $=1$ in comparison to uncrosslinked polymer brushes but increases for 
$\mathrm{LS}=2$ and $\mathrm{LS}=4$, which can also be explained as before for the case of NS $=50$ crosslinkers. The shear stress increases with increasing length of crosslinkers (LS) at all speeds studied. The coefficient of friction decreases with increasing length of crosslinkers at all speeds studied and at LS $=4$ the coefficients of friction at all speeds are close to those for the uncrosslinked polymer-brush systems. The density profiles (Figure 6(d)) show that the brush height increases with increasing length of crosslinkers, and the density profile for LS $=4$ is quite similar to that of the uncrosslinked polymer brush system. As the length of crosslinkers increases, the crosslinked polymer brushes behaved more and more like uncrosslinked polymer brushes and assist in brush-mediated lubrication[10, 11, 18]. For systems with shorter crosslinkers, as can be seen from the density profile, fewer polymer chains are available at the free end of grafted polymers to facilitate brush assisted lubrication and this results in higher friction, as we observe. Compared to systems with NS $=50$ crosslinkers, the effect of length of crosslinkers is more clearly visible in systems with NS = 200 crosslinkers.

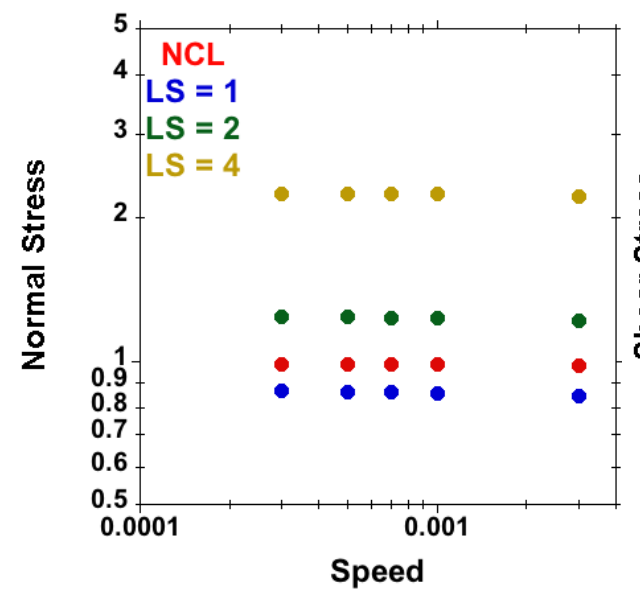

(a)

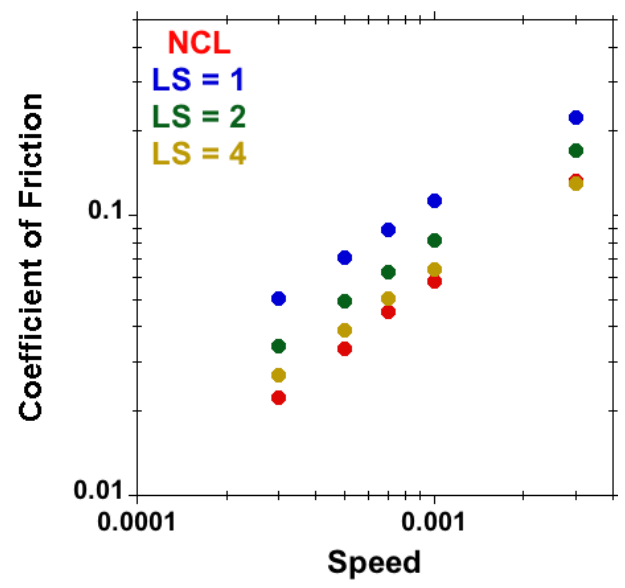

(c)

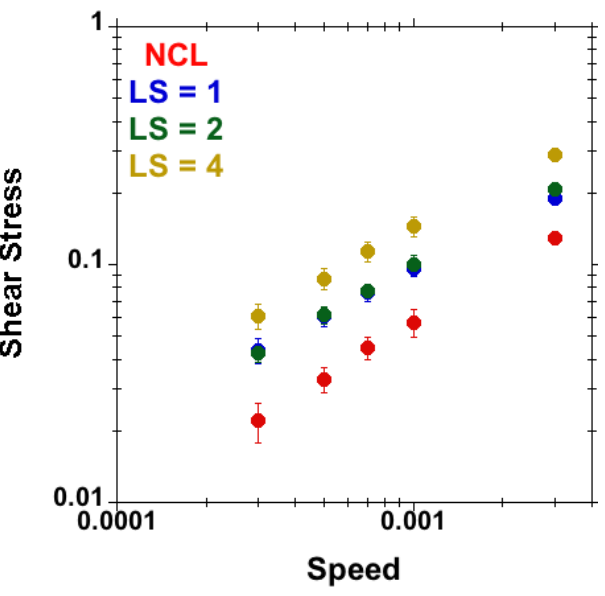

(b)

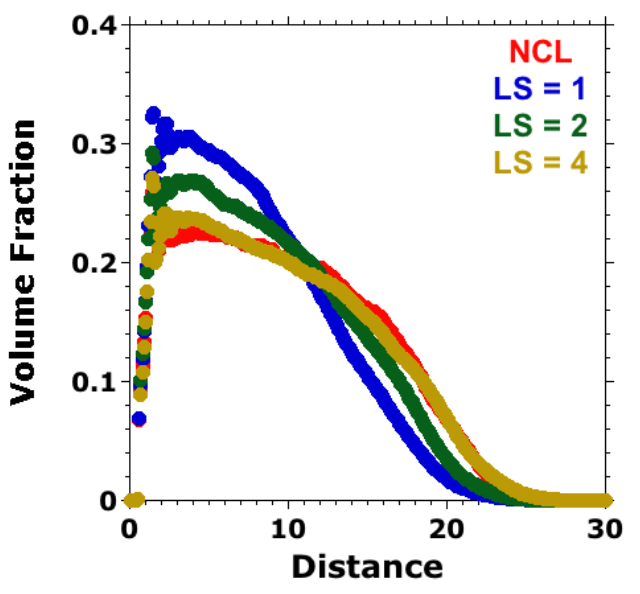

(d)

Figure 6: Effect of length of crosslinkers at constant degree/number of crosslinkers, NS = 200. (a) Normal stress, (b) shear stress, (c) coefficient of friction, all against speed and, (d) volume fraction against distance to the grafting surface for brush systems with $M=50, N=50, \rho=0.075, \mathrm{NS}=200$ and for different lengths of crosslinker chains (LS) $=0$ (NCL), 1, 2 and 4 . The volume fraction profile is shown at separation $D=30$ between grafting surface and wall, at vanishing speed, $S=0$. 


\section{CONCLUSIONS}

Crosslinked polymer brushes were modeled using a coarse-grained MD technique. The tribological behavior of crosslinked polymer brushes under shear has been compared to that of uncrosslinked polymer brushes. The simulations were performed at fixed separation, subject to different shear speeds of the tethered beads, using an implicit solvent. In general the coefficient of friction for all the crosslinked systems was found to be larger than that for the uncrosslinked polymer brushes. We observed an increase in the coefficient of friction with increasing degree of crosslinking and a decrease in the coefficient of friction with increasing length of crosslinkers for all speed-dependent studies. Crosslinking affects the tribological behavior in two ways: (i) as the degree of crosslinking increases there are fewer polymer chains in the outer layer to take part in brush-assisted lubrication, and (ii) crosslinked polymer chains offer more resistance to shear than non-crosslinked ones.

We have used a simple approach to study the effect of crosslinking on the tribological behavior of polymer brushes. This work can be extended by performing studies over a wider range of degree of crosslinking for various lengths of crosslinkers, to gain an enhanced quantitative understanding of the influence of the length of crosslinkers on the mechanical behavior of gels under shear. We are not aware of existing experimental data of sufficiently well characterized crosslinked brushes that could be used to test all predictions of this model. We are, however, in the process of generating such data.

\section{ACKNOWLEDGEMENT}

The authors are thankful to ETH Research Commission for financial support to carry out the work.

\section{REFERNCES}

1. Napper, D.H.: Polymeric stabilization of colloidal dispersions. London ; New York : Academic Press (1983).

2. Hucknall, A., Simnick, A.J., Hill, R.T., Chilkoti, A., Garcia, A., Johannes, M.S., Clark, R.L., Zauscher, S., Ratner, B.D.: Versatile synthesis and micropatterning of nonfouling polymer brushes on the wafer scale. Biointerphases. 4, FA50-7 (2009).

3. Auroy, P., Auvray, L., Leger, L.: Characterization of the Brush Regime for Grafted Polymer Layers at the Solid-Liquid Interface. Phys. Rev. Lett. 66, 719-722 (1991).

4. Lee, S., Spencer, N.D., Erdemir, A., Martin, J.M.: Achieving ultralow friction by aqueous, brush-assisted lubrication. Superlubricity. 365-396 (2007).

5. Li, A.: Structure-property Relationships of Surface-grafted Polymeric Architectures: From Ultra-Thin Films To Quasi-3D Polymer Assemblies. PhD Thesis. ETH Zurich (2013).

6. Dunn, A.C., Sawyer, W.G., Angelini, T.E.: Gemini Interfaces in Aqueous Lubrication with Hydrogels. Tribol. Lett. 54, 59-66 (2014).

7. Freeman, M.E., Furey, M.J., Love, B.J., Hampton, J.M.: Friction, wear, and lubrication of hydrogels as synthetic articular cartilage. Wear. 241, 129-135 (2000).

8. Irfachsyad, D., Tildesley, D., Malfreyt, P.: Dissipative particle dynamics simulation of grafted polymer brushes under shear. Phys. Chem. Chem. Phys. 4, 3008-3015 (2002).

9. Chen, M., Briscoe, W.H., Armes, S.P., Klein, J.: Lubrication at Physiological Pressures by Polyzwitterionic Brushes. Science. 323, 1698-1701 (2009).

10. Klein, J., Perahia, D., Warburg, S.: Forces between polymer-bearing surfaces undergoing shear. Nature. 352, (1991).

11. Li, A., Benetti, E.M., Tranchida, D., Clasohm, J.N., Schönherr, H., Spencer, N.D.: SurfaceGrafted, Covalently Cross-Linked Hydrogel Brushes with Tunable Interfacial and Bulk Properties. Macromolecules. 44, 5344-5351 (2011).

12. Gong, J.P.: Friction and lubrication of hydrogels?its richness and complexity. Soft Matter. 2, 544 (2006). 
13. Stachowiak, G.W., Batchelor, A.W.: Engineering tribology. Elsevier, Amsterdam. London. New York. Tokyo (1993).

14. Raviv, U., Klein, J.: Adhesion, Friction, and Lubrication between Polymer-Bearing Surfaces. In: Polymer Science: A Comprehensive Reference. pp. 607-628. Elsevier (2012).

15. Klein, J., Kumacheva, E., Perahia, D., Mahalu, D., Warburg, S.: Interfacial sliding of polymerbearing surfaces. Faraday Discuss. 98, 173 (1994).

16. Klein, J., Kumacheva, E., Mahaiu, D., Perahia, D., Fetters, L.J.: Reduction of frictional forces between solid surfaces bearing polymer brushes. Nature. 370, 634-636 (1994).

17. Nalam, P.C., Ramakrishna, S.N., Espinosa-Marzal, R.M., Spencer, N.D.: Exploring Lubrication Regimes at the Nanoscale: Nanotribological Characterization of Silica and Polymer Brushes in Viscous Solvents. Langmuir. 29, 10149-10158 (2013).

18. Lee, S., Spencer, N.D.: Sweet, Hairy, Soft, and Slippery. Science. 319, 575-576 (2008).

19. Kreer, T.: Polymer-brush lubrication: A review of recent theoretical advances. Soft Matter. 12(15), 3479-3501 (2016).

20. Grest, G.S.: Computer simulations of shear and friction between polymer brushes. Curr. Opin. Colloid Interface Sci. 2, 271-277 (1997).

21. Hoy, R.S., Grest, G.S.: Entanglements of an End-Grafted Polymer Brush in a Polymeric Matrix. Macromolecules. 40, 8389-8395 (2007).

22. Grest, G.S.: Grafted polymer brushes: a constant surface pressure molecular dynamics simulation. Macromolecules. 27, 418-426 (1994).

23. Kremer, K., Grest, G.S.: Dynamics of entangled linear polymer melts: A molecular-dynamics simulation. J. Chem. Phys. 92, 5057-5086 (1990).

24. Grest, G.: Interfacial Sliding of Polymer Brushes: A Molecular Dynamics Simulation. Phys. Rev. Lett. 76, 4979-4982 (1996).

25. Murat, M., Grest, G.S.: Molecular dynamics simulations of the force between a polymer brush and an AFM tip. Macromolecules. 29, 8282-8284 (1996).

26. Grest, G.S.: Normal and shear forces between polymer brushes. Adv. Polym. Sci. 138, 149183 (1999).

27. Singh, M.K., Ilg, P., Espinosa-Marzal, R.M., Kröger, M., Spencer, N.D.: Polymer Brushes under Shear: Molecular Dynamics Simulations Compared to Experiments. Langmuir. 31, 4798-4805 (2015).

28. Galuschko, A., Spirin, L., Kreer, T., Johner, A., Pastorino, C., Wittmer, J., Baschnagel, J.: Frictional Forces between Strongly Compressed, Nonentangled Polymer Brushes: Molecular Dynamics Simulations and Scaling Theory. Langmuir. 26, 6418-6429 (2010).

29. Nalam, P.C.: Polymer Brushes in Aqueous Solvent Mixtures: Impact of Polymer Conformation on Tribological Properties. PhD Thesis. ETH Zurich. (2012).

30. Kim, S.H., Opdahl, A., Marmo, C., Somorjai, G.A.: AFM and SFG studies of pHEMA-based hydrogel contact lens surfaces in saline solution: adhesion, friction, and the presence of noncrosslinked polymer chains at the surface. Biomaterials. 23, 1657-1666 (2002).

31. Pan, Y.-S., Xiong, D.-S., Ma, R.-Y.: A study on the friction properties of poly(vinyl alcohol) hydrogel as articular cartilage against titanium alloy. Wear. 262, 1021-1025 (2006).

32. Gong, J.P., Higa, M., Iwasaki, Y., Katsuyama, Y., Osada, Y.: Friction of gels. Journal of Physical Chemistry B. 101, 5487-5489 (1997).

33. Caravia, L., Dowson, D., Fisher, J., Corkhill, P.H., Tighe, B.J.: Friction of Hydrogel and Polyurethane Elastic Layers When Sliding Against Each Other Under a Mixed Lubrication Regime. Wear. 181, 236-240 (1995).

34. Gong, J.P., Kurokawa, T., Narita, T., Kagata, G., Osada, Y., Nishimura, G., Kinjo, M.: Synthesis of Hydrogels with Extremely Low Surface Friction. J. Am. Chem. Soc. 123, 55825583 (2001).

35. Mamada, K., Fridrici, V., Kosukegawa, H., Kapsa, P., Ohta, M.: Friction Properties of Poly(vinyl alcohol) Hydrogel: Effects of Degree of Polymerization and Saponification Value. Tribol. Lett. 42, 241-251 (2011).

36. Bavaresco, V.P., Zavaglia, C.A.C., Reis, M.C., Gomes, J.R.: Study on the tribological properties of pHEMA hydrogels for use in artificial articular cartilage. Wear. 265, 269-277 (2008).

37. Ohsedo, Y., Takashina, R., Gong, J.P., Osada, Y.: Surface friction of hydrogels with welldefined polyelectrolyte brushes. Langmuir. 20, 6549-6555 (2004).

38. Ishikawa, Y., Hiratsuka, K.-I., Sasada, T.: Role of water in the lubrication of hydrogel. Wear. 261, 500-504 (2005).

39. Alexander, S.: Adsorption of chain molecules with a polar head a scaling description. J. Phys- 
Paris. 38, 983-987 (1977).

40. De Gennes, P.G.: Conformations of Polymers Attached to an Interface. Macromolecules. 13, 1069-1075 (1980).

41. Marko, J.F., Chakrabarti, A.: Static and dynamic collective correlations of polymer brushes. Phys. Rev. E. 48, 2739-2743 (1993).

42. Milner, S.T., Witten, T.A., Cates, M.E.: Theory of the grafted polymer brush. Macromolecules. 21, 2610-2619 (1988).

43. Zhulina, Y.B., Pryamitsyn, V.A., Borisov, O.V.: Structure and conformational transitions in grafted polymer chain layers. A new theory. Polymer Science U.S.S.R. 31, 205-216 (1989).

44. Binder, K., Müller, M.: Monte Carlo simulation of block copolymers. Curr. Opin. Colloid Interface Sci. 5, 315-323 (2000).

45. Hsu, H.-P., Paul, W.: A fast Monte Carlo algorithm for studying bottle-brush polymers. Comput. Phys. Commun. 182, 2115-2121 (2011).

46. Binder, K., Müller, M., Schmid, F., Werner, A.: Interfaces in partly compatible polymer mixtures: a Monte-Carlo simulation approach. Physica A. 249, 293-300 (1998).

47. Doyle, P.S., Shaqfeh, E., Gast, A.P.: Rheology of polymer brushes: A Brownian dynamics study. Macromolecules. 31, 5474-5486 (1998).

48. Goujon, F., Ghoufi, A., Malfreyt, P., Tildesley, D.J.: The kinetic friction coefficient of neutral and charged polymer brushes. Soft Matter. 9, 2966 (2013).

49. Carrillo, J.-M.Y., Brown, W.M., Dobrynin, A.V.: Explicit Solvent Simulations of Friction between Brush Layers of Charged and Neutral Bottle-Brush Macromolecules. Macromolecules. 45, 8880-8891 (2012).

50. Elliott, I.G., Kuhl, T.L., Faller, R.: Compression of High Grafting Density Opposing Polymer Brushes Using Molecular Dynamics Simulations in Explicit Solvent. J. Phys. Chem. B. 117, 4134-4141 (2013).

51. Jentzsch, C., Sommer, J.-U.: Polymer brushes in explicit poor solvents studied using a new variant of the bond fluctuation model. J. Chem. Phys. 141, 104908 (2014).

52. Kreer, T., Binder, K., Müser, M.H.: Friction between Polymer Brushes in Good Solvent Conditions: Steady-State Sliding versus Transient Behavior. Langmuir. 19, 7551-7559 (2003).

53. Kreer, T., Müser, M.H., Binder, K., Klein, J.: Frictional Drag Mechanisms between PolymerBearing Surfaces. Langmuir. 17, 7804-7813 (2001).

54. Kreer, T., Müser, M.H.: On the tribology and rheology of polymer brushes in good solvent conditions: a molecular dynamics study. Wear. 254, 827-831 (2003).

55. de Beer, S., Müser, M.H.: Alternative dissipation mechanisms and the effect of the solvent in friction between polymer brushes on rough surfaces. Soft Matter. 9, 7234 (2013).

56. Dimitrov, D.I., Milchev, A., Binder, K.: Polymer brushes in solvents of variable quality: Molecular dynamics simulations using explicit solvent. J. Chem. Phys. 127, 084905 (2007).

57. Galuschko, A.: Molecular Dynamics Simulations of Sheared Polymer Brushes. PhD Thesis. Strasbourg University. (2010).

58. Pastorino, C., Kreer, T., Müller, M., Binder, K.: Comparison of dissipative particle dynamics and Langevin thermostats for out-of-equilibrium simulations of polymeric systems. Phys. Rev. E. 76, 026706 (2007).

59. Chen, J., Zhou, S.-M., Ma, B.-G., Zhang, L.-M., Yi, J.-Z.: Molecular dynamics simulations on dextran hydrogels. E-Polymers. 13, 1-8 (2013).

60. Walter, J., Sehrt, J., Vrabec, J., Hasse, H.: Molecular Dynamics and Experimental Study of Conformation Change of Poly( N-isopropylacrylamide) Hydrogels in Mixtures of Water and Methanol. J. Phys. Chem. B. 116, 5251-5259 (2012).

61. Tönsing, T., Oldiges, C.: Molecular dynamic simulation study on structure of water in crosslinked poly(N-isopropylacrylamide) hydrogels. Phys. Chem. Chem. Phys. 3, 5542-5549 (2001).

62. Hoffmann, M., Lang, M., Sommer, J.-U.: Gelation threshold of cross-linked polymer brushes. Phys. Rev. E. 83, 021803 (2011).

63. Ou, X., Han, Q., Dai, H.-H., Wang, J.: Molecular dynamic simulations of the water absorbency of hydrogels. J Mol Model. 21, 231-10 (2015).

64. Plimpton, S.: Fast Parallel Algorithms for Short-Range Molecular Dynamics. J. Comput. Phys. 117, 1-19 (1995).

65. Kröger, M.: Efficient hybrid algorithm for the dynamic creation of wormlike chains in solutions, brushes, melts and glasses. Comput. Phys. Commun. 118, 278-298 (1999). 